\title{
EDIFICATE
}

I Congreso de Escuelas de Edificación y Arquitectura Técnica de España València, 4 y 5 de noviembre de 2021

Escuela Técnica Superior de Ingeniería de Edificación

Universitat Politècnica de València

Doi: https://doi.org/10.4995/EDIFICATE2021.2021.13564

\section{Radiografía del Grado en Arquitectura Técnica de la EPS de Zamora (Universidad de Salamanca)}

\section{Radiography of the technical architecture bachelor of Zamora EPS (Salamanca University)}

\section{Ascensión Rodríguez-Esteban¹, M. Almudena Frechilla-Alonso², Ana Belén González-Rogado 3 y Ana Belén Ramos-Gavilán ${ }^{4}$}

1Universidad de Salamanca, mare@usal.es, ${ }^{2}$ Universidad de Salamanca, almudena.frechilla@usal.es, ${ }^{3}$ Universidad de Salamanca, abg@usal.es, ㄴuniversidad de Salamanca, aramos@usal.es

\section{Abstract}

The Degree in Technical Architecture (GAT) of the Higher Polytechnic School of Zamora, of the University of Salamanca, is going through a difficult moment in terms of the number of enrollments, with a drastic reduction, which began in the 2009-2010 academic year and which It bottomed out in the 2014-2015 academic year, a situation from which, to date, it has not yet recovered. So much so, that in the current course does not reach $10 \%$ of which had its high points. This Degree, which replaced the Technical Architecture degree in the 2009-2010 academic year, began to be taught in 1998, in a modern and attractive University Campus that had just opened in Zamora, in which other Engineering Degrees were offered and that he had a great tug in his first ten years. There is no doubt that it was the crisis of the "brick" that caused that fall, but having recovered, the situation does not improve. Given this fact, we propose to make an X-ray of the current state of the GAT, putting white on black a series of parameters that can shed some light on the reasons why the number of registrations does not rebound.

Keywords: Technical Architecture Degree, Higher Polytechnic School of Zamora, University of Salamanca, current situation, number of enrollments, student satisfaction.

\section{Resumen}

El Grado de Arquitectura Técnica (GAT) de la Escuela Politécnica Superior de Zamora, de la Universidad de Salamanca, está pasando por un momento difícil en cuanto al número de matriculaciones, con una reducción drástica, que 
comenzó en el curso 2009-2010 y que tocó fondo en el curso 2014-2015, situación de la que, a día de hoy, todavía no se ha recuperado. Tal es así, que en el curso actual no llega al $10 \%$ de la que tuvo en sus momentos álgidos. Este Grado, que sustituyó a la titulación de Arquitectura Técnica en el curso 2009-2010, comenzó a impartirse en el año 1998, en un Campus Universitario que acababa de inaugurarse en Zamora, moderno y atractivo, en el que se ofertaban otros Grados de Ingeniería y que tuvo un gran tirón en sus primeros diez años. No cabe duda de que fue la crisis del "ladrillo" la que propició esa caída, pero habiéndose recuperado, la situación no mejora. Ante este hecho, nos proponemos hacer una radiografía del estado actual del GAT, poniendo blanco sobre negro a una serie de parámetros que puedan arrojar alguna luz sobre los motivos por los cuales el número de matriculaciones no repunta.

Palabras clave: Grado Arquitectura Técnica, Escuela Politécnica Superior de Zamora, Universidad de Salamanca, situación actual, número de matrículas, satisfacción estudiantes. 


\section{Introducción}

El Grado de Arquitectura Técnica (GAT) de la Universidad de Salamanca se imparte en la Escuela Politécnica Superior de Zamora (EPSZ), junto a otros 6 Grados y 2 Dobles Grados de ingenierías, que comparten profesorado e instalaciones. Se enclava en el Campus Viriato, que se construyó entre los años 1997 y 1999 sobre el antiguo Cuartel de Caballería homónimo, uno de los conjuntos edificatorios más emblemáticos de la ciudad (Ávila de la Torre, 2005) (Fig. 1).

La adaptación de las antiguas instalaciones castrenses para uso docente proporcionó unas magníficas infraestructuras en pleno ensanche urbano, cerca de las estaciones de autobuses y de ferrocarril y también de los dos hospitales. La proximidad a estos equipamientos y servicios no es una cuestión menor, puesto que puede influir en la cuestión que se pone sobre la mesa en esta comunicación, al igual que el hecho de que en el mismo Campus se encuentren la Escuela Universitaria de Magisterio y la Escuela Universitaria de Enfermería, ya que permite hacer una comparativa respecto a ítems comunes que se van a mostrar y que pueden ayudar a esclarecer el estudio.
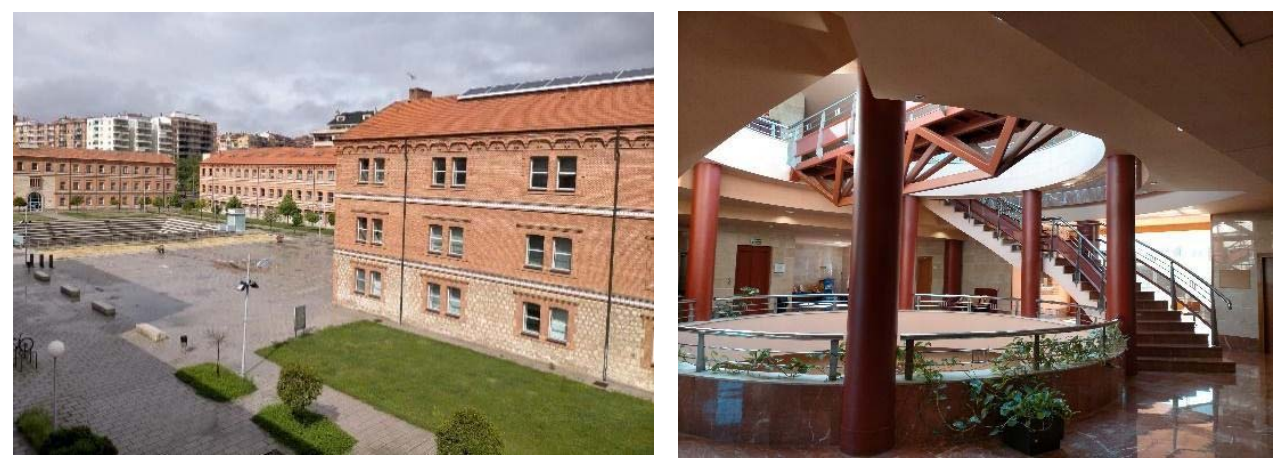

Fig. 1 Imágenes del Campus Viriato, vista general y espacio interior del edificio de la EPSZ

Desde que la titulación de Arquitectura Técnica se incorporó a la EPS de Zamora en 1998, y tras su transformación en Grado de Arquitectura Técnica en 2009, estos estudios gozaron de una demanda de estudiantes extraordinaria, hasta el punto de que en el curso 2005-2006 se amplió la oferta de matrículas en primer curso, de 90 a 120 (Fig. 2). Fue a partir de la crisis provocada por la burbuja inmobiliaria, cuando el número empezó a descender sin que, hasta el momento, haya llegado la recuperación esperada (Campos 2008). Este hecho resulta altamente preocupante para la comunidad universitaria y para Zamora en general, ya que el Campus Universitario es un revulsivo para atraer población y dinamizar la actividad a nivel local e, incluso, regional. Tal es así, que su promoción se lleva a cabo de forma conjunta entre la Universidad de Salamanca y el Ayuntamiento de la ciudad. 


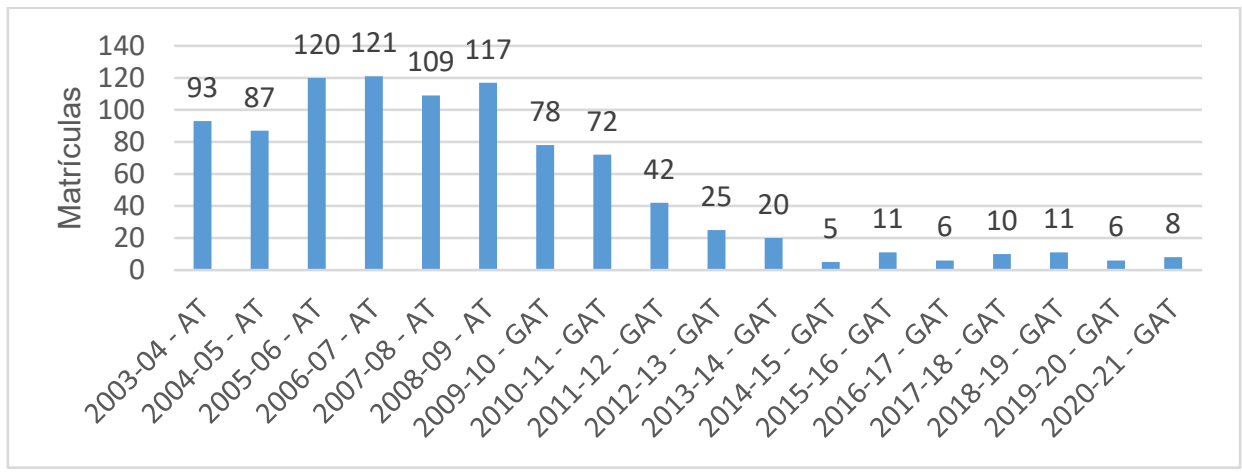

Fuente: Elaboración propia

Fig. 2 Estudiantes matriculados en primer curso de Arquitectura Técnica. Evolución

Los grados que se imparten en la EPS de Zamora tienen un comportamiento dispar frente a la demanda, siendo el Grado de Ingeniería Civil (GIC) el que se más se asemeja al GAT, incluso su declive es más pronunciado, frente al Grado de Ingeniería Mecánica (GIM) que se va manteniendo, aunque ha sufrido un ligero descenso (Fig. 3). Junto a estos Grados está el Grado de Ingeniería de Informática en Sistemas de Información (GIISI), el Grado de Ingeniería Agroalimentaria (GIA), el Grado de Ingeniería de Materiales (GIMAT), y los dobles Grados de Ingeniería de Materiales e Ingeniería Mecánica (GIMM) y de Ingeniería Informática en Sistemas de Información e Información y Documentación (GIID).

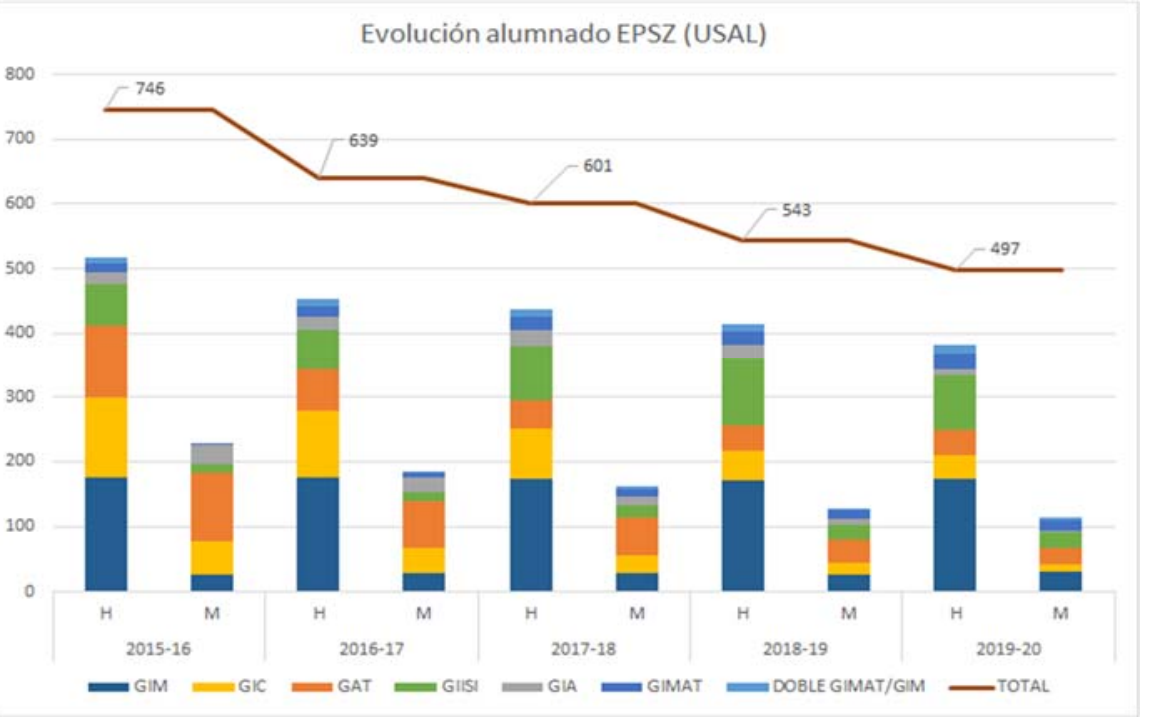

Fuente: Elaboración propia

Fig. 3 Evolución del alumnado por género y comparación del número de matrículas en la EPSZ entre Grados 
A esto se añaden los datos sobre matriculaciones en primer curso en diversos Grados del Campus Viriato y su evolución en los últimos 10 años, así como el número de plazas ofertadas. Para ello, extraemos los datos de tres Grados habilitantes representativos de la EPSZ (GIC, GAT, GIM), y los comparamos con los otros dos Grados que se imparten en las otras Escuelas del Campus: el Grado en Maestro en Educación Primaria (GMEP) y el Grado en Enfermería (GE) (Fig. 4).

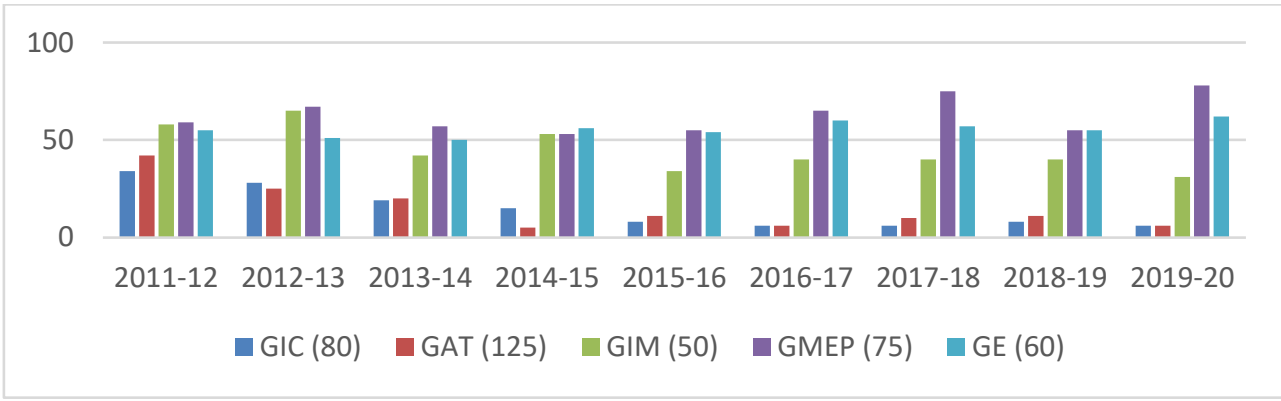

Fuente: Elaboración propia

Fig. 4 Comparación del número de matrículas del GAT, GIC, GIM, GMEP y GE. Entre paréntesis, el número de plazas ofertadas

Por otra parte, hay que destacar que, en el GAT, el porcentaje de mujeres es muy alto, máxime si lo comparamos con el GIM que se imparte en el Campus de Zamora (Fig. 5). Además, en los últimos cursos ha aumentado la presencia de estudiantes extranjeros que vienen de Marruecos y de China.

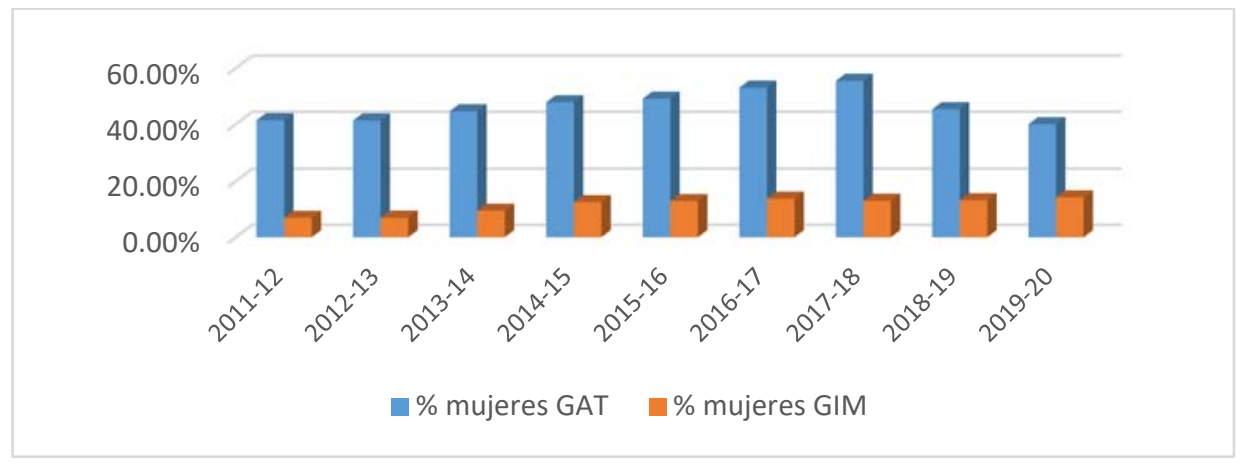

Fuente: Elaboración propia

Fig. 5 Comparación del porcentaje de mujeres matriculadas en el GAT y en el GIM

Con esta información de partida, se va a realizar una exposición de los resultados obtenidos en los ítems, que nos arrojan información sobre el estado de la cuestión y su evolución desde diez años atrás. Para ello, consideramos importante conocer el grado de satisfacción de los estudiantes con la Actividad Docente del profesorado y con el Programa Formativo, así como la inserción laboral de los egresados. 
Además, y considerando que el GAT es un Grado habilitante, es fundamental hacer un barrido por la categoría del personal docente, dado que hay muchas materias que necesitan un perfil profesional muy vinculado a ellas (COLIN 2018)

\section{Objetivos}

El objetivo de este estudio es hacer una radiografía general del Grado en Arquitectura Técnica de la EPSZ, para conocer, mediante el análisis de diversos datos y encuestas publicados, los aspectos que pudieran influir en la baja tasa de matrícula, comparándolos con ítems similares de otros Grados ubicados en el Campus de Zamora. Así mismo, aprovechando este foro, se pretende impulsar una mesa de conversación donde se exponga la situación de otros Centros de dimensiones similares.

\section{Desarrollo. Estado de la cuestión}

Consideramos que la información más importante es la que nos aportan los estudiantes, por ese motivo, es fundamental conocer su tasa de rendimiento, así como los resultados de las encuestas de satisfacción que han realizado sobre la Actividad Docente del Profesorado y sobre el Programa Formativo, sin olvidarnos de la opinión que tienen los egresados y su Inserción Laboral. Otros dos aspectos que pueden arrojar información y que también exponemos son las relaciones institucionales con las empresas y con otras universidades.

\subsection{Grado de satisfacción de los estudiantes con la Actividad Docente del Profesorado}

Cada dos años, los estudiantes responden anónimamente a una encuesta sobre el grado de satisfacción con la actividad docente del profesorado, atendiendo a las siguientes preguntas (tabla 1), donde se muestran los datos estadísticos descriptivos respecto al conjunto de ítems en porcentajes y cinco rangos de respuesta: TDA: totalmente de acuerdo, DA: de acuerdo, IN: indiferente, ED: en desacuerdo, TED: totalmente en desacuerdo.

Tabla 1. Preguntas a los estudiantes y respuestas en \% (curso 2018-2019)

\begin{tabular}{|c|c|c|c|c|c|}
\hline PREGUNTAS & TED & ED & IN & $\overline{\mathrm{DA}}$ & TDA \\
\hline 1. El / la profesor/a explica con claridad & 1.11 & 2.77 & 11.51 & 42.66 & 37.95 \\
\hline 2. Resuelve las dudas plateadas y orienta a los estudiantes & 1.11 & 4.72 & 11.94 & 38.61 & 43.61 \\
\hline 3. Organiza y estructura bien las actividades que realizamos & 0.56 & 5.32 & 19.05 & 35.29 & 39.78 \\
\hline 4. Las actividades son provechosas para lograr los objetivos & 0.84 & 3.62 & 13.93 & 39.28 & 42.34 \\
\hline $\begin{array}{l}\text { 5.Favorece la participación del estudiante en el desarrollo de la } \\
\text { actividad }\end{array}$ & 1.96 & 6.98 & 18.16 & 35.47 & 37.43 \\
\hline 6.Está accesible para ser consultado por los estudiantes & 1.69 & 2.82 & 13.80 & 36.62 & 45.07 \\
\hline $\begin{array}{l}\text { 7. Ha facilitado mi aprendizaje, conocimientos habilidades y } \\
\text { destrezas }\end{array}$ & 1.39 & 5.29 & 17.55 & 38.44 & 37.33 \\
\hline 8. Los recursos didácticos utilizados son adecuados & 1.96 & 6.42 & 15.36 & 40.78 & 35.47 \\
\hline 9. La bibliografía y los materiales didácticos facilitados son útiles & 2.25 & 7.04 & 20.28 & 34.37 & 36.06 \\
\hline $\begin{array}{l}\text { 10.Los métodos de evaluación se corresponden con el desarrollo } \\
\text { docente }\end{array}$ & 1.53 & 2.14 & 11.93 & 41.59 & 42.81 \\
\hline
\end{tabular}




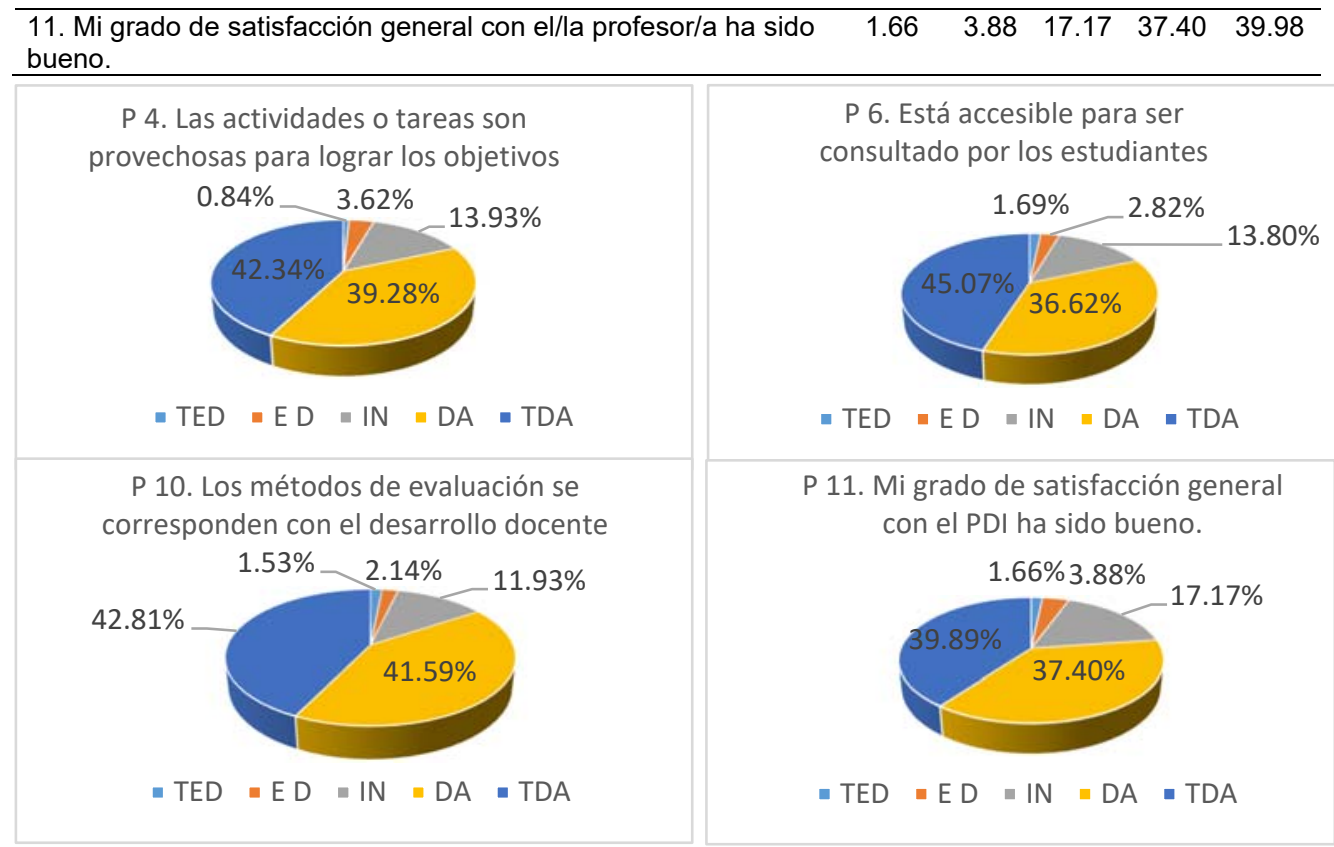

Fuente: Elaboración propia

Fig. 6 Respuestas a las preguntas 4, 6, 10 y 11

\subsection{Grado de satisfacción de los estudiantes con el Programa Formativo}

La opinión de los estudiantes respecto al Programa Formativo se extrae de una encuesta que se divide en cinco bloques: BLOQUE 1. Plan de Estudios y su Estructura; BLOQUE 2. Organización de la enseñanza; BLOQUE 3. Proceso de enseñanza - aprendizaje; BLOQUE 4. Instalaciones e infraestructuras y BLOQUE 5. Acceso y atención al alumno. (DOC USAL).

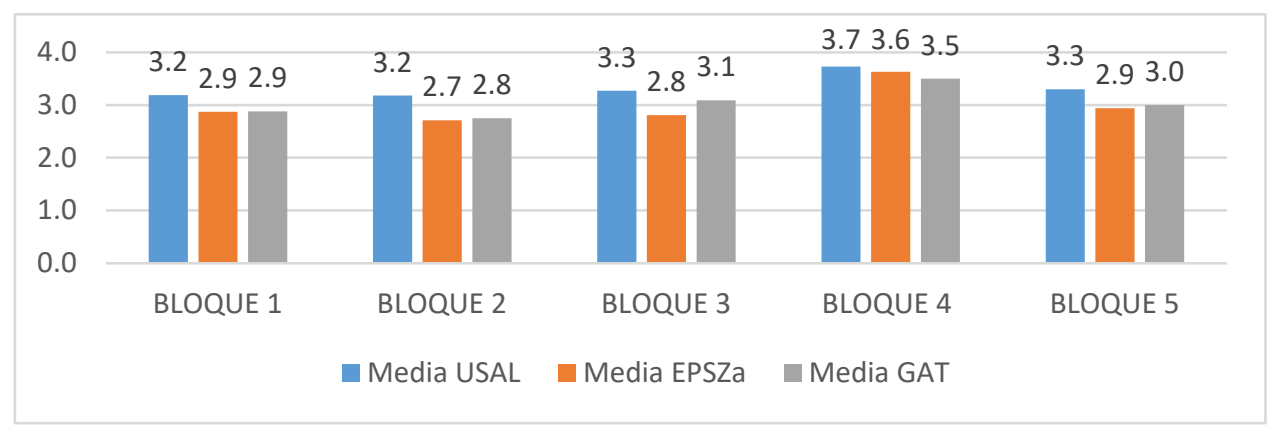

Fuente: Elaboración propia

Fig. 7 Respuestas de la encuesta de satisfacción de los estudiantes con el Programa Formativo, sobre 5 puntos. Curso 2019-2020 


\subsection{Tasa de rendimiento}

La Tasa de rendimiento, como relación porcentual entre el número de créditos superados y el número de créditos matriculados por titulación y curso académico, ofrece una lectura fundamental sobre el funcionamiento del Grado, informando sobre la facilidad o dificultad que tienen los estudiantes en aprobar las asignaturas de las que se matriculan. Con el fin de establecer el rango y comprobar si es un motivo del descenso de estudiantes, la comparamos con la tasa de rendimiento del GIM, que es el Grado habilitante de la EPSZ que mejor se mantiene (Fig. 8).

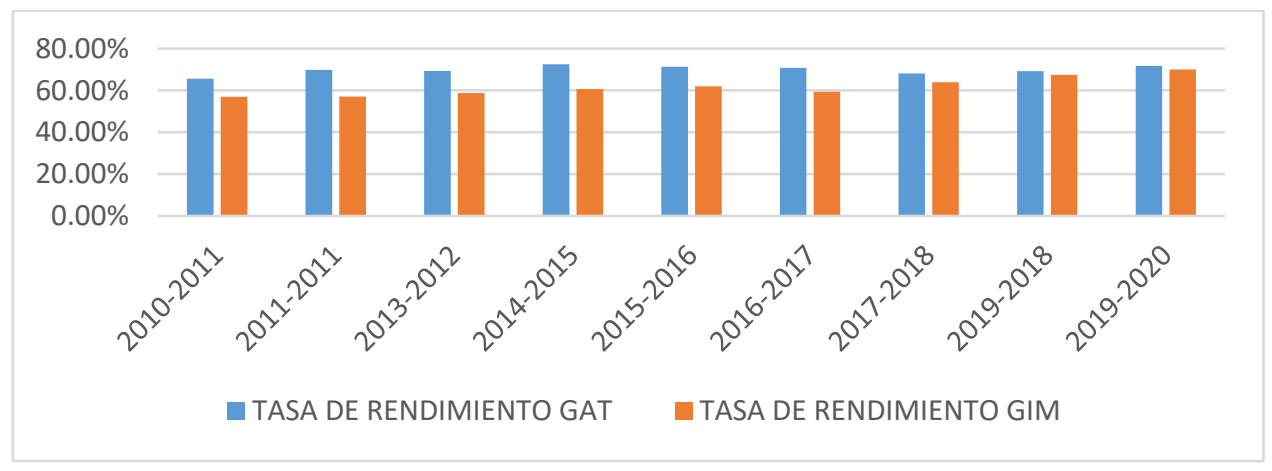

Fig. 8 Tasa de rendimiento del GAT y del GIM

Fuente: Elaboración propia

\subsection{Valoración de los egresados}

Sabiendo que la tendencia de los jóvenes a la hora de elegir el Grado depende en gran medida de las posibilidades que tenga para encontrar trabajo, las respuestas que nos interesan de los egresados son aquellas vinculadas con su satisfacción con el Grado que estudiaron (Fig. 8) y con su situación laboral (Fig. 9). (DOC EGRESADOS)

La encuesta corresponde a la promoción 2017-18 y se envió en el año 2021 a una población de 34 , participando el $26,47 \%$ de las personas a las que se les solicitó, porcentaje poco representativo pero que se acerca mucho al global de la Universidad, que está en un 27,49\%. 
M. A. Rodríguez-Esteban, M. A. Frechilla-Alonso, A. B. González-Rogado, A. B. Ramos-Gavilán
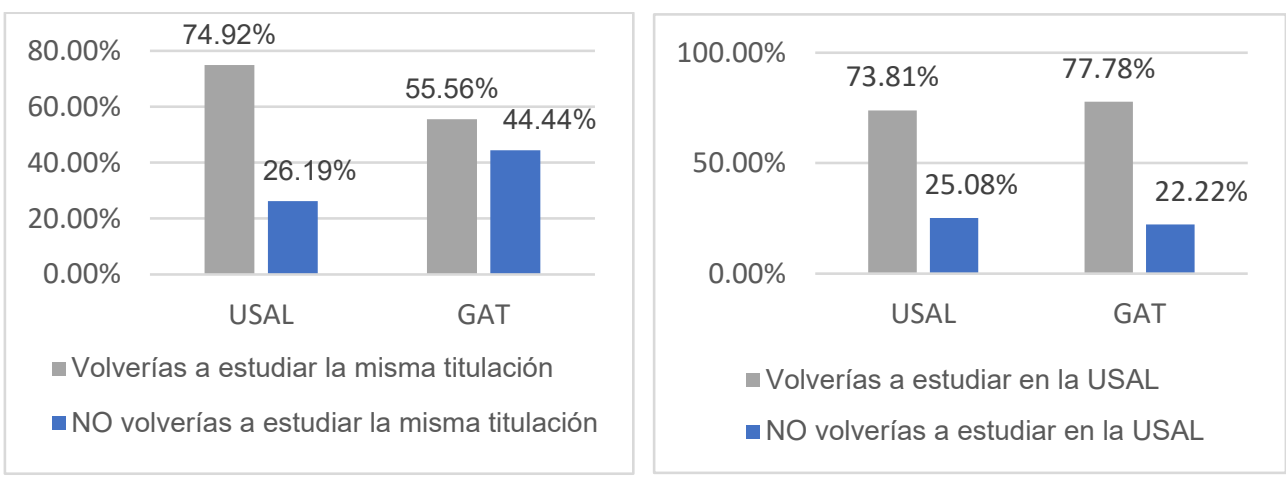

Fig. 9 Situación laboral de los egresados

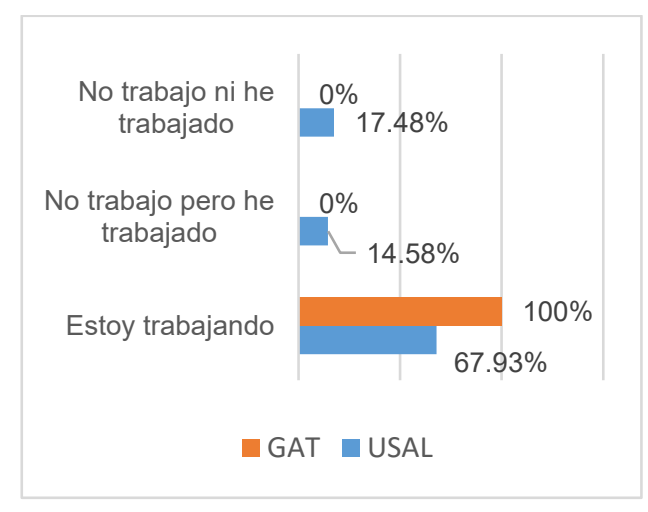

Fig. 10 Respuestas sobre la situación laboral de los egresados

\subsection{Perfil del Personal Docente}

Considerando que el Grado en Arquitectura a Técnica es un Grado habilitante, y que tiene un número importante de asignaturas que se pueden considerar profesionalizantes, llamadas así a aquellas que tienen aplicación directa en el campo profesional, cabe valorar la existencia de profesores asociados que pudieran acercar a las aulas el conocimiento que han adquirido y siguen adquiriendo por su experiencia laboral. 


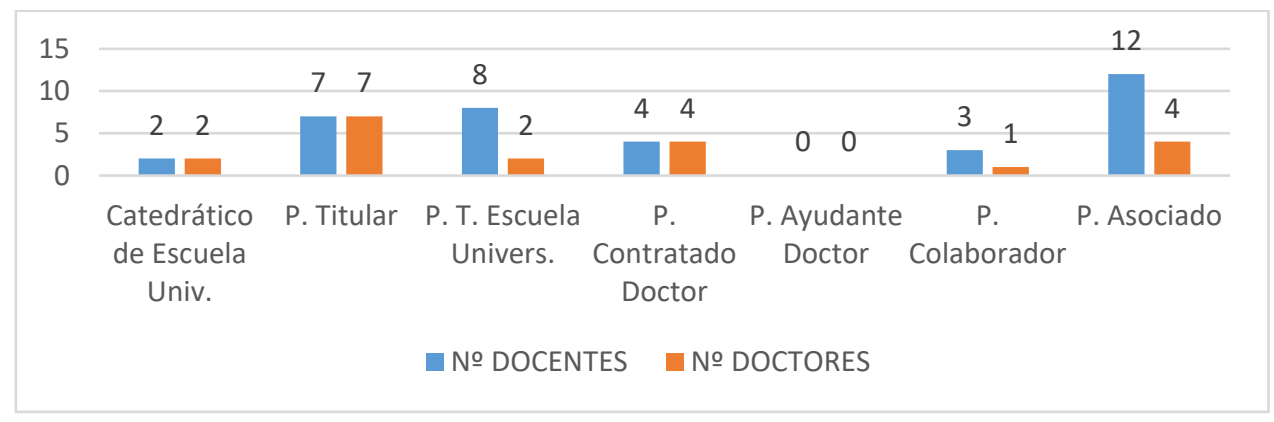

Fuente: Elaboración propia

Fig. 11 Número de docentes por su categoría profesional y $n^{\circ}$ de doctores. Curso 2019-20

\subsection{Relación con el sector empresarial}

Este ítem está relacionado directamente con la asignatura optativa Prácticas de Empresa (PE) (6 ECTS), en la cual, los estudiantes deben realizar prácticas curriculares en una empresa, durante 150 horas. Así mismo, aquellos que hayan superado esta asignatura y deseen continuar con prácticas, pueden realizar prácticas extracurriculares durante un máximo de 6 meses y 900 horas.

Para poder realizarse, la guía académica del GAT establece que: La Escuela Politécnica Superior de Zamora facilitará a los alumnos una serie de empresas del sector de la titulación con las que previamente el Centro habrá establecido un convenio de colaboración. Entre estas empresas los alumnos harán su elección. Todos los convenios llevan incorporados un tutor por parte de la Empresa y un tutor por parte de la Universidad. Para ello, los estudiantes que están matriculados de la asignatura PE tienen acceso a un listado en el que figuran 40 empresas, de las cuales 7 están vigentes en la fecha actual, 21 no están no vigentes y 11 en tramitación.

Cabe decir que la asignatura optativa "Prácticas de Empresa" es elegida por prácticamente el $100 \%$ de los estudiantes del GAT.

\subsection{Movilidad de estudiantes}

Este aspecto es relevante en la medida que se observan las relaciones que tiene la EPSZ con otros centros para el intercambio de estudiantes en todos los Grados que se imparten en Zamora, con el programa ERASMUS y el programa SICUE (Fig. 10). En concreto, para el GAT, hay convenios para dos estudiantes con la Universidad del Salento, en Lecce (Italia) y otros dos con la Universidad de Lisboa (Portugal). 


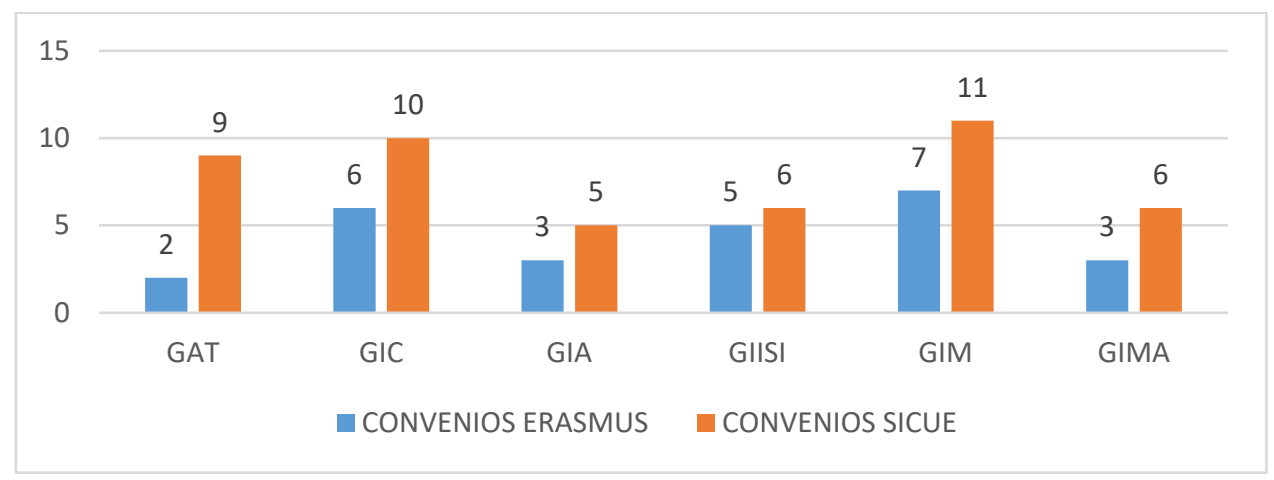

Fuente: Elaboración propia

Fig. 12 Número de Convenios ERASMUS + en los Grados de la EPSZ. Curso 2020-2021

\section{Resultados}

A tenor de la tendencia de números de matrículas en la EPSZ, los resultados arrojan datos poco optimistas en general y preocupantes, en particular para el GAT. Sin embargo, y comparado con el GIM, es un Grado atractivo para las mujeres, con una tasa de matrícula femenina entre el $40 \%$ y el $50 \%$.

Sin embargo, el descenso de matrículas contrasta con tres datos fundamentales: el grado de satisfacción del estudiante con la actividad docente del profesorado, la cual, en todos los ítems la puntuación supera el 3,5 sobre 5 puntos. De hecho, entre el $36 \%$ y el $42 \%$ están de acuerdo con la actividad y en ese mismo rango se mueven los que opinan que están totalmente de acuerdo. Esto frente a valores inferiores al $2 \%$ que opinan estar totalmente en desacuerdo.

Respecto al Programa Formativo, en todos los bloques preguntados, la valoración se encuentra entorno al 3,0, alcanzando 3,5 sobre 5,0 puntos, cuando valoran las instalaciones y son ligeramente inferiores a la media de puntuación de la USAL y de la EPSZ,

La Tasa de rendimiento del GAT lleva una tendencia más o menos constante durante los diez últimos años, con valores cercanos al 70\%, llegando a su mejor dato en el curso 2014-2015, que superó el $72 \%$. No obstante, estos porcentajes son mejores que los del GIM, que no han alcanzado el $70 \%$ en ningún curso de los analizados.

Otro aspecto que podría preocupar es la tasa de inserción laboral, pero que, a tenor de la encuesta realizada a los egresados, en el caso del GAT, está en el $100 \%$. Sin embargo, esto contrasta con su intención de volver a estudiar lo mismo, ya que un $22 \%$ no lo confiesa que no lo haría.

Respecto a las circunstancias propias del GAT, como Grado habilitante, es importante conocer la ratio de Profesorado Asociado con respecto al total. En este sentido, el 33\% de la plantilla tiene esta categoría, aunque es importante destacar que el $33 \%$ de ella es Doctor, mérito necesario para optar a otra categoría más académica que profesional. Aparte, en el 
GAT de la EPSZ, el profesorado con dedicación completa que imparte las materias relacionadas con la vida profesional del Arquitecto Técnico tiene relación laboral con la construcción, al amparo del art. 83 de la Ley Orgánica de Universidades (LOU).

En cuanto a las empresas, el número que tienen convenio con la USAL para acoger estudiantes en prácticas es muy limitado. Además, no se tiene conocimiento de que haya otro tipo de relación Universidad-Empresa en este campo como sucede en otras universidades, que han creado sinergias bidireccionales (Otero 2018).

Por último, las posibilidades sobre la movilidad del alumnado a otras universidades son muy reducidas, ya que solo existe convenio con dos, y ninguna es de lengua inglesa. Cabe decir que el número de estudiantes que solicitan movilidad SICUE y Erasmus representa un porcentaje muy elevado, respecto al total de matriculados.

Respecto a los aspectos donde hay debilidades, la Escuela contempla un Plan de Mejoras, en el autoinforme de renovación de la acreditación, de 2016, con propuestas concretas para solventarlas.

\section{Conclusiones}

De todo el estudio expuesto, se concluye que la actividad docente del profesorado y el Programa Formativo no son aspectos que, a priori, influyan en la baja tasa de matrícula. Tampoco lo es el perfil del profesorado, altamente relacionado con la construcción, ni tampoco, a día de hoy, la inserción laboral.

Así mismo, un aspecto tan importante como es la tasa de rendimiento, que se sitúa en valores superiores a los de otros Grados de la Escuela que tienen mayor matrícula, tampoco debería ser un hándicap para atraer estudiantes a la EPSZ.

En el lado opuesto están las relaciones institucionales con las empresas y con otras universidades, que se antojan escasas y poco atractivas para el alumnado.

De todo el análisis realizado no es posible extraer una conclusión certera sobre la problemática que se plantea, siendo posible que el foco de la cuestión gire en torno a otros aspectos ajenos al Grado que habría que analizar con detenimiento en otro estudio, además de observar e investigar las actuaciones de otros centros de tamaño similar al de Zamora, donde se imparta el Grado de Arquitectura Técnica o de Ingeniería de la Construcción, que tengan un mayor éxito en tasa de matrícula.

\section{Referencias}

ÁVILA DE LA TORRE, A (2010). Arquitectura y Urbanismo en Zamora (1850-1950). Zamora: Instituto de Estudios Zamoranos Florián de Ocampo.

CAMPOS ECHEVARRIA, J. L. (2008): La burbuja inmobiliaria española. Madrid: Marcial Pons.

COLIN RIVERA, M, I. (2018): “Importancia del perfil docente”. ACTA EDUCATIVA Universidad Abierta Revista. $\mathrm{N}^{\circ}$ 20. https://revista.universidadabierta.edu.mx/publicacion-20/ [Consulta: 16 de junio de 2021]. 
ESCUELA POLITÉCNICA SUPERIOR DE ZAMORA (2021). Grado en Arquitectura Técnica. $<$ https://www.usal.es/grado-en-arquitectura-tecnica > [Consulta: 18 de junio de 2021] [sin autoría reconocida].

ESCUELA POLITÉCNICA SUPERIOR DE ZAMORA (2021). Grado en Arquitectura Técnica. $<$ https://poliz.usal.es/politecnica/v1 r00/?m=Grados\&it=7\&doc=grados/Renovacion_acreditacion_GA T.pdf> [Consulta: 12 de junio de 2021] [sin autoría reconocida].

ESCUELA POLITÉCNICA SUPERIOR DE ZAMORA (2021). Prácticas externas, Programas de Movilidad, Normativas <https:// poliz.usal.es/politecnica/v1r00/> [Consulta: 8 de junio de 2021] [sin autoría reconocida].

ESCUELA POLITÉCNICA SUPERIOR DE ZAMORA (2021). Grado en Ingeniería Mecánica. $<$ https://www.usal.es/grado-en-ingenieria-mecanica-eps-de-zamora> [Consulta: 8 de junio de 2021] [sin autoría reconocida]

ESCUELA POLITÉCNICA SUPERIOR DE ZAMORA (2021). Grado en Ingeniería Agroalimentaria. <https://www.usal.es/grado-en-ingenieria-agroalimentaria-zamora> [Consulta: 8 de junio de 2021] [sin autoría reconocida]

ESCUELA POLITÉCNICA SUPERIOR DE ZAMORA (2021). Grado en Ingeniería Civil <https://www.usal.es/grado-en-ingenieria-civil-eps-de-zamora> [Consulta: 8 de junio de 2021] [sin autoría reconocida]

ESCUELA POLITÉCNICA SUPERIOR DE ZAMORA (2021). Grado en Ingeniería Informática en Sistemas de Información. <https://www.usal.es/grado-en-ingenieria-informatica-en-sistemas-deinformacion> [Consulta: 8 de junio de 2021] [sin autoría reconocida]

ESCUELA POLITÉCNICA SUPERIOR DE ZAMORA (2021). Grado en Ingeniería de Materiales <https://www.usal.es/grado-en-ingenieria-materiales> [Consulta: 8 de junio de 2021] [sin autoría reconocida]

ESCUELA UNIVERSITARIA DE MAGISTERIO DE ZAMORA (2021). <https://www.usal.es/escuelauniversitaria-de-magisterio-de-zamora> [Consulta: 9 de junio de 2021] [sin autoría reconocida]

ESCUELA UNIVERSITARIA DE ENFERMERÍA DE ZAMORA (2021) <https://www.usal.es/ grado-enenfermeria-eu-de-enfermeria-de-zamora-centro-adscrito> [Consulta: 9 de junio de 2021] [sin autoría reconocida].

OTERO-MOLINA, M. (2018): "Sinergia bidireccional Universidad-Empresa. Caso de estudio: Aula Universitaria de Arquitectura" en XXVI Congreso Universitario de Innovación Educativa de las Enseñanzas Técnicas. Oviedo. Disponible https://digibuo.uniovi.es/dspace/handle/10651/47930. [Consulta: 7 de junio de 2021].

UNIVERSIDAD DE SALAMANCA (2021) Relaciones internacionales <https://relint.usal.es/es/proyectos/erasmus> [Consulta: 9 de junio de 2021] [sin autoría reconocida]

UNIVERSIDAD MAYOR DE SAN ANDRÉS (2021) GRADO EN INGENIERÍA MECÁNICA <https:// mecanica.umsa.edu.bo/?page_id=131\#page-content> [Consulta: 15 de junio de 2021] [sin autoría reconocida]. 